\title{
EGF promotes mammalian cell growth by suppressing cellular senescence
}

Cell Research (2015) 25:135-138. doi:10.1038/cr.2014.141; published online 4 November 2014

\section{Dear Editor,}

Cellular senescence is a state of irreversible proliferative arrest that is believed to be an important determinant of both cancer development and organismal aging [1]. Although numerous molecules have been found to promote senescence, little is known about the nature of anti-senescent factors under physiological conditions. To identify factors that may control the cellular senescent process, we cultured human mammary epithelial (HME) or human bronchial epithelial (HBE) cells in either complete growth medium, or medium formulated to lack individual hormones, growth factors, and other supplements commonly used in primary cell culture. Whereas most culture conditions had no noticeable effect on cell growth, one-week incubation in medium deprived of epidermal growth factor (EGF) resulted in cells displaying an enlarged, flattened morphology with obvious growth suppression. Although EGF is routinely used as a general mitogen in cell culture experiments to drive the proliferation of myriad cell types that are otherwise recalcitrant to growth, a direct role for EGF in suppressing senescence in normal mammalian cells has not been previously documented. Further analysis of HME cells cultured in the absence of EGF revealed that these cells displayed high SA- $\beta$-gal activity in addition to severely impaired proliferation (Figure 1A and 1B). Immunoblotting analysis of senescence markers demonstrated that these cells exhibited increased levels of p21 protein and diminished phosphorylation of the Rb tumor suppressor (Figure 1C), whereas expression of p16 remained undetectable after EGF depletion. Elevated SA- $\beta$-gal activity was also observed in additional human primary cell types cultured without EGF, including aortic smooth muscle, microvascular endothelial, umbilical vein endothelial, and prostate epithelial cells (Supplementary information, Figure S1A). These results suggest that normal human cells grown in culture require EGF in order to escape senescence and sustain proliferation.

Recognizing the known involvement of cellular senescence in normal development and aging, we next sought to determine whether EGF might be involved in the regulation of mammalian stem cell activity in culture. Although certain types of stem cells are known to undergo differentiation in response to this growth factor, EGF is commonly added exogenously to maintain the self-renewal of murine neural stem cells (NSCs) [2]. To test whether EGF suppresses senescence in this context, we cultured NSCs derived from newborn pups in complete NSC medium or in medium lacking EGF. As was the case for the differentiated human cell types, NSCs exposed for 3 days to medium lacking EGF became senescent, as evidenced by smaller colony size, reduced proliferative ability, and elevated SA- $\beta$-gal activity (Supplementary information, Figure S1B-S1E), suggesting that EGF acts as an anti-senescent factor to prevent senescence of NSCs and maintain their self-renewal capability.

To further study EGF's ability to regulate senescence, we treated normal human cells with increasing concentrations of the chemical inhibitor erlotinib, which is an ATP analog that selectively blocks ATP hydrolysis at the EGF receptor (EGFR) kinase active site. Upon 3 days of culture in the presence of erlotinib, HME and HBE cells appeared morphologically similar to those deprived of EGF, failed to proliferate, and contained high SA- $\beta$-gal activity (Figure 1D and 1E, Supplementary information, Figure S1F and S1G), indicating that EGF suppresses senescence by activating EGFR in these cell types. To quantify the level of senescence induced by EGFR inhibition, we made use of $\mathrm{C}_{12} \mathrm{FDG}$, a substrate that becomes fluorescent after SA- $\beta$-gal-catalyzed cleavage [3]. Incubation with $\mathrm{C}_{12} \mathrm{FDG}$ and subsequent analysis by flow cytometry revealed that $\sim 70 \%$ of HME and HBE cells were senescent after two days of treatment with $1 \mu \mathrm{M}$ erlotinib (Figure 1F-1H and Supplementary information, Figure S1H-S1J). Four additional primary cell types also appeared to senesce when cultured in the presence of erlotinib (Supplementary information, Figure S1K).

Senescent cells are known to secrete a large number of cytokines, chemokines, regulatory growth factors, proteases, and extracellular matrix proteins, a pro-inflammato- 


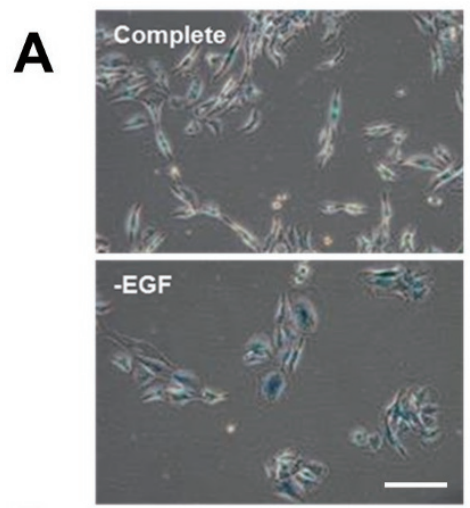

D

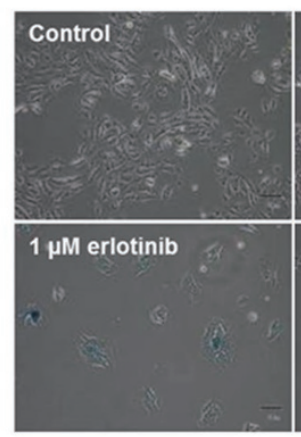

$\mathbf{F}$

G
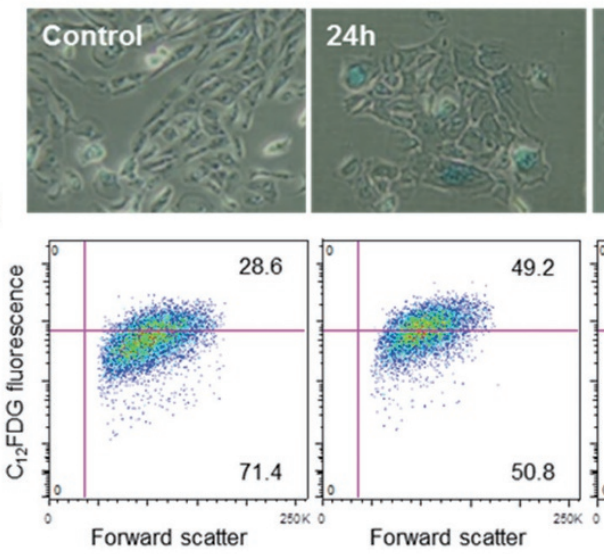

I
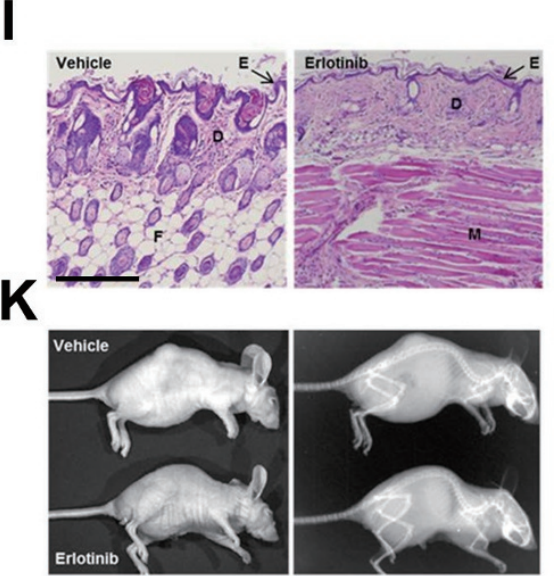
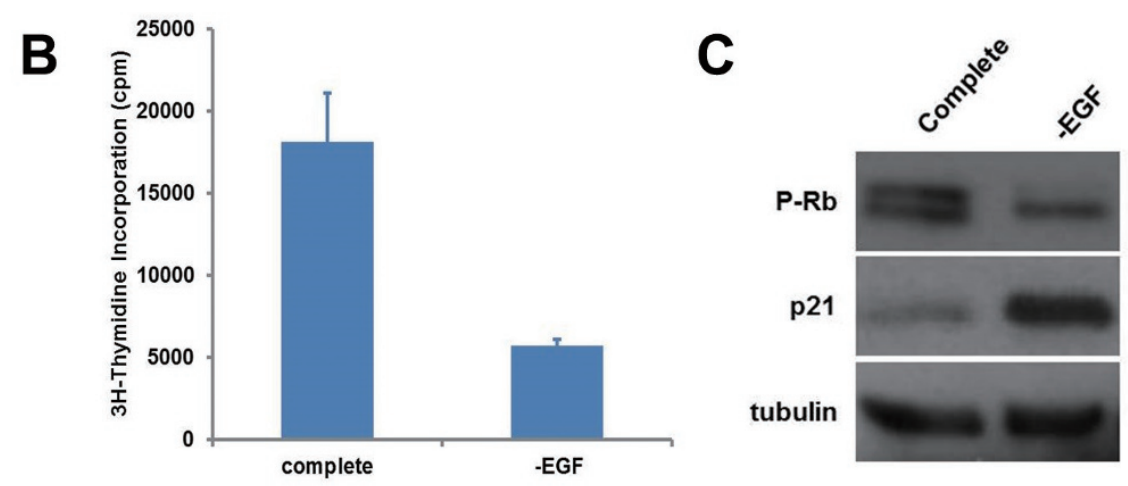

E

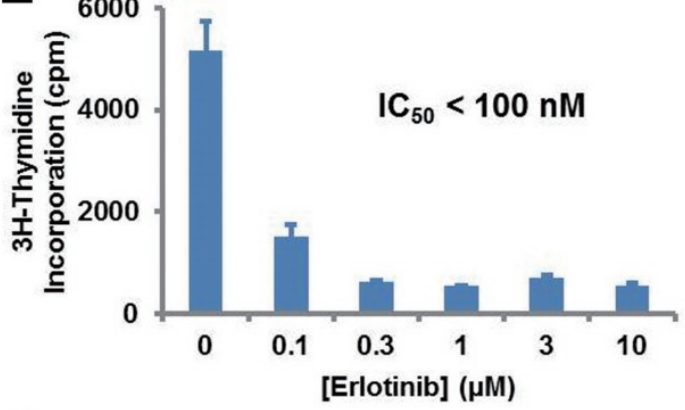

H

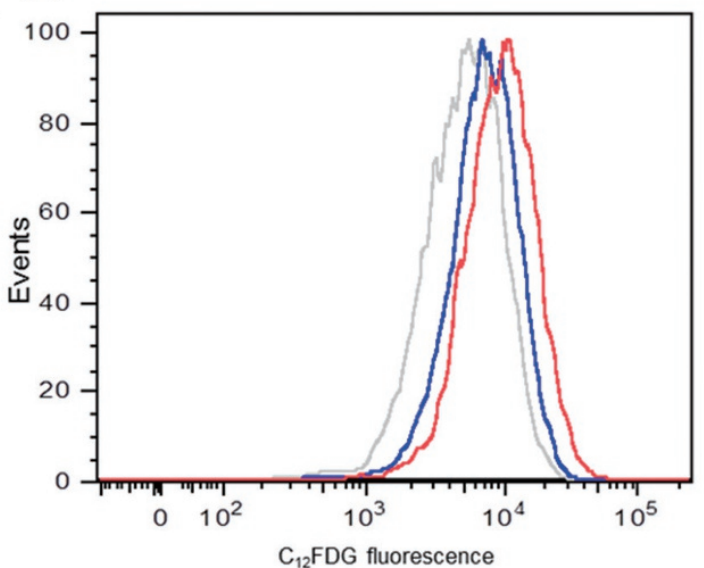

M

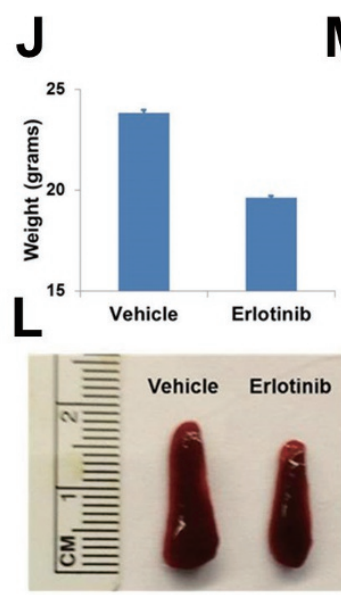

Skin

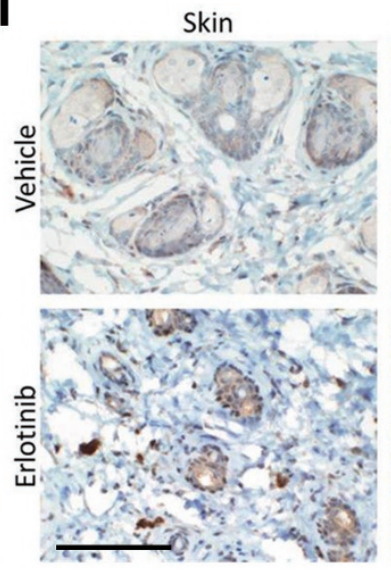

Spleen

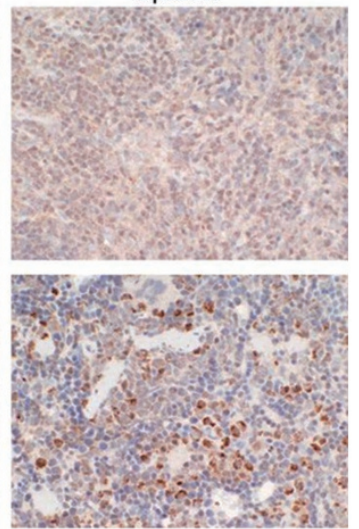


ry phenotype known as the senescence-associated secretory phenotype (SASP) [4]. To determine whether EGFR inhibition produces a SASP, we measured the mRNA expression profile of 31 genes which represent a collection of SASP-associated products known to be induced in different cell types during either replicative or oncogene-induced senescence [4]. As shown in Supplementary information, Figure S1L, 72 h treatment of HBE cells with $1 \mu \mathrm{M}$ erlotinib resulted in a significant upregulation of 19 out of the 31 SASP-associated factors. This widespread upregulation of many SASP-associated factors in erlotinib-treated HBE cells provides further evidence that a lack of EGFR signaling results in a cell state exhibiting many hallmarks of cellular senescence.

Since erlotinib and other pharmacological agents targeting EGFR, such as gefitinib, are currently used to treat certain types of cancer, we next tested the effect of systemic EGFR inhibition on normal mammalian physiology using immunodeficient BALB/c mice. Interestingly, daily erlotinib administration via oral gavage produced pronounced anatomical deficiencies in several mouse tissues. First, upon sustained erlotinib administration many of the mice developed dry, peeling skin. Hematoxylin and eosin staining of skin from these animals revealed that those treated with erlotinib had thinner epidermal and dermal layers and lacked the subcutaneous fat seen in their vehicle-treated counterparts (Figure 1I). Similar skin defects have been previously reported in both EGFR conditional knockout mice as well as in cancer patients receiving EGFR inhibitor therapy [5], indicating that this skin phenotype is very likely to result from the selective inhibition of EGFR kinase activity rather than to be an off-target effect of the drug.

In addition to skin atrophy, it was apparent that erlotinib-treated mice exhibited dramatic weight loss and kyphosis (Figure $1 \mathrm{~J}$ and $1 \mathrm{~K}$ ), phenotypic features that have been observed in mouse models of premature aging [6]. Furthermore, upon dissection we noticed marked organ atrophy as evidenced by reduced spleen size (Figure 1L) and a lack of lymphocyte-containing white pulp. Supporting a role for cellular senescence in the development of this phenotype, immunohistochemical analysis of skin and spleen from erlotinib-treated animals revealed elevated expression of the cell cycle inhibitor p16 in both tissue types (Figure 1M). Recent studies indicate that the murine immune system is able to recognize and clear senescent cells to limit disease progression [7]. To test whether the observed phenotype was related to the lack of a fully functional immune system in the BALB/c nude mice, we treated immunocompetent $\mathrm{C} 57 \mathrm{BL} / 6$ mice with erlotinib using the same schedule and dose as treating $\mathrm{BALB} / \mathrm{c}$ mice. Interestingly, the $\mathrm{C} 57 \mathrm{BL} / 6$ mice were as sensitive to EGFR inhibition as the BALB/c nude mice, and upon phenotypic analysis it was determined that the immunocompetent animals showed similar levels of weight loss, skin and splenic atrophy to their immunodeficient counterparts (Supplementary information, Figure S1M-S1Q).

Together, this spectrum of abnormalities suggests that sustained erlotinib administration in mice produces a phenotype in some tissues resembling premature aging. Since EGFR inhibition is shown here to induce senes-

Figure 1 Anti-senescence function of EGF in human epithelial cells and mouse tissues. (A-C) EGF depletion results in cellular senescence of HME cells. HME cells were exposed for 1 week to complete culture medium or to medium lacking EGF. Cells cultured in the absence of EGF displayed an enlarged morphology, elevated SA- $\beta$-gal activity (A), decreased proliferation (B), and reduced $\mathrm{Rb}$ phosphorylation and elevated p21 expression (C), all markers that are commonly associated with cellular senescence. (D-H) EGFR pharmacological inhibition causes cellular senescence of HME cells. In D and E, HME cells were treated for three days with complete culture medium supplemented with increasing concentrations of erlotinib. Cells were then stained for SA- $\beta$-gal activity (D), or metabolically labeled with $2 \mu \mathrm{Ci}$ of $\left[{ }^{3} \mathrm{H}\right]$-thymidine for $16 \mathrm{~h}$ (E). Progressively decreasing amounts of label incorporation were observed for cells cultured in the presence of erlotinib. Experiments were performed in triplicate; error bars indicate SD. In F-H, quantification of erlotinib-induced cellular senescence of HME cells using flow cytometry. HME cells were exposed to $1 \mu \mathrm{M}$ erlotinib for 24 or $48 \mathrm{~h}$ and then analyzed for SA- $\beta$-gal activity using either a chromogenic assay $(\mathbf{F})$ or a fluorescence-based assay ( $\mathbf{G}$ and $\mathbf{H}$, see Supplementary information, Data S1). Both assay types indicated that SA- $\beta$-gal reached maximal activity from 24 to $48 \mathrm{~h}$ after treatment. (A, D, F) Scale bar, $50 \mu \mathrm{m}$. (I-M) Mice treated with erlotinib display early aging-associated phenotypes. Skin atrophy in erlotinib-treated mice. BALB/c nude mice (five per group) were treated with either vehicle or erlotinib (50 mg/kg/day) for 4 weeks. Skin was fixed in $4 \%$ PFA, stained using $\mathrm{H} \& \mathrm{E}$, and photographed at 10x magnification. Note the reduced epidermal thickness and fat content resulting from EGFR inhibition. E, epidermis; D, dermis; F, fat; M, muscle. Scale bar, $250 \mu \mathrm{m}$. (I). Reduced weight of mice treated with daily erlotinib. Error bars indicate SD (J). Representative photograph and X-ray of tumor-bearing BALB/c nude mice treated with vehicle or erlotinib. Note the reduced size, kyphosis, and generally aged appearance of the erlotinib-treated mouse (K). Spleen atrophy in erlotinib-treated mice (see also Supplementary information, Figure S1P-S1Q) (L). Immunohistochemical staining of skin and spleen tissue from vehicle- or erlotinib-treated mice was performed using an antibody specific for $\mathrm{p} 16$. Images shown were photographed at $40 \times$. Scale bar, $100 \mu \mathrm{m}(\mathbf{M})$. 
cence in a variety of normal cell types, we propose that this syndrome could have resulted from widespread senescence throughout the organism. This would be in line with increasing evidence indicating an association between cellular senescence and mammalian aging [8]. Both skin atrophy and organ dysfunction have been observed in lung cancer patients undergoing EGFR inhibitor therapy [9]. It will be important to determine whether these are independently occurring side effects or are part of a general program of accelerated aging in these individuals, with the aim of maximizing the therapeutic benefit of EGFR inhibition while causing minimal damage to normal cells and tissues.

In conclusion, this study uncovers a previously unknown, but fundamentally important function for EGF as a potent factor controlling the cell fate of normal mammalian cell types. Long regarded as merely a mitogen, EGF may also regulate cellular and tissue homeostasis via its anti-senescence activity. What might be the non-pathological setting for the senescent state resulting from EGF depletion? Recent research has demonstrated that in the mouse embryo cellular senescence is required for the proper development of the inner ear, mesonephros, apical epidermal ridge, neural roof plate, and syncytiotrophoblast [10-12]. In considering the finding that EGF plays an anti-senescent role in certain stem and differentiated cell types, we propose that an absence of EGF or other growth factors might be responsible for generating this senescent state within the mammalian embryo. Indeed, several commonalities appear to exist between developmentally programmed and EGF depletion-induced senescence. Both appear to be natural processes not associated with any overt stress conditions. In addition, the senescent markers detected in both states are similar: elevation of $\mathrm{p} 21$ expression and absence of DNA damage. Finally, developmental senescence was shown to be regulated by phosphoinositide 3-kinase (PI3K) signaling [10], which is one of the major path- ways downstream of EGFR. While these similarities are intriguing, more research will be required to determine what relationship exists, if any, between developmentally programmed and EGF depletion-induced senescence.

\section{Acknowledgments}

We thank Su Z and the Duke University Department of Pathology for assistance with immunohistochemistry and H\&E staining; Markowitz G, Zhang Y, and Counter C for helpful scientific input. This work was supported by National Institutes of Health (CA154586 to XFW and CA059365 to PBA).

Peter B Alexander ${ }^{1}$, Lifeng Yuan ${ }^{1}$, Pengyuan Yang ${ }^{1}$, Tao Sun ${ }^{1}$, Rui Chen ${ }^{1}$, Handan Xiang ${ }^{1}$, Jiekai Chen ${ }^{2}$, Haoyu $\mathrm{Wu}^{2}$, Daniel R Radiloff ${ }^{3}$, Xiao-Fan Wang ${ }^{1}$

${ }^{I}$ Department of Pharmacology and Cancer Biology, Duke University Medical Center, Durham, NC 27710, USA; ' Guangzhou Institutes of Biomedicine and Health, Chinese Academy of Sciences, Guangzhou, Guangdong 510530, China; ${ }^{3}$ Department of Pediatrics, University of British Columbia, Vancouver, V6T 1Z4, Canada

Correspondence: Xiao-Fan Wang

E-mail: xiao.fan.wang@duke.edu

\section{References}

1 Kuilman T, Michaloglou C, Mooi WJ, et al. Genes Dev 2010; 24:24632479.

2 Tropepe V, Sibilia M, Ciruna BG, et al. Dev Biol 1999; 208:166-188.

3 Debacq-Chainiaux F, Erusalimsky JD, Campisi J, et al. Nat Protoc 2009; 4:1798-1806.

4 Coppe JP, Patil CK, Rodier F, et al. PLoS Biol 2008; 6:2853-2868.

5 Roberts RB, Arteaga CL, Threadgill DW. Cancer Cell 2004; 5:115-120.

6 Kuro-o M, Matsumura Y, Aizawa H, et al. Nature 1997; 390:45-51.

7 Kang TW, Yevsa T, Woller N, et al. Nature 2011; 479:547-551.

8 Baker DJ, Wijshake T, Tchkonia T, et al. Nature 2011; 479:232-236.

9 Ren S, Li Y, Li W, et al. Respiration 2012; 84:431-435.

10 Munoz-Espin D, Canamero M, Maraver A, et al. Cell 2013; 155:11041118.

11 Storer M, Mas A, Robert-Moreno A, et al. Cell 2013; 155:1119-1130.

12 Chuprin A, Gal H, Biron-Shental T, et al. Genes Dev 2013; 27:23562366.

(Supplementary information is linked to the online version of the paper on the Cell Research website.) 